\title{
Lipid composition of stomach oil in a procellariiform seabird Puffinus tenuirostris: implications for food web studies
}

\author{
Maëlle Connan ${ }^{1,2}$, Patrick Mayzaud ${ }^{1, *}$, Marc Boutoute $^{1}$, Henri Weimerskirch $^{2}$, \\ Yves Cherel ${ }^{2}$
}

\author{
${ }^{1}$ Observatoire Océanologique, Océanographie Biochimique et Ecologie, Laboratoire d'Océanographie de \\ Villefranche-sur-Mer - Unité mixte de recherche 7093, BP 28, 06234 Villefranche-sur-Mer, France \\ ${ }^{2}$ Centre d'Etudes Biologiques de Chizé - Unité propre de recherche du Centre National de la Recherche Scientifique, \\ BP 14, 79360 Villiers-en-Bois, France
}

\begin{abstract}
Some procellariiform seabirds use a dual strategy for provisioning their chicks by alternating between short and long foraging trips (LT). Trophic relationships of adult birds are unknown when they feed for themselves during LT because digestion processes preclude direct prey determination. Since stomach contents collected after LT contain oil of dietary origin, we tested the use of oil lipids as prey trophic markers using the Tasmanian short-tailed shearwater Puffinus tenuirostris as a model seabird. The intra-specific variability of stomach oils was investigated through lipid class composition, and their fatty acid and fatty alcohol profiles. Oils mainly consisted of wax esters (WE) and triacylglycerols (TAG) (49 to 86 and 7 to $41 \%$, respectively). Major fatty acids of TAG were in a decreasing order 18:1n-9, 16:0, 16:1n-7, 14:0, 20:5n-3 and 22:6n-3. The WE-fatty acid profiles were dominated by $18: 1 \mathrm{n}-9$ and $16: 1 \mathrm{n}-7$ while fatty alcohol profiles were dominated by 16:0. Fatty alcohol and fatty acid patterns were tested as possible descriptors of ingested prey (derived from literature data) through multivariate discriminant analyses. Comparisons of the WE fatty alcohol patterns showed a close association with the alcohol structure of 3 myctophid fish species namely Krefftichthys anderssoni, Gymnoscopelus braueri and Electrona antarctica; these results were corroborated by WE fatty acid analysis. Comparison of TAG fatty acid patterns showed the highest similarity between oils and the digestive gland of the myctophid-eater squid Moroteuthis ingens in association with the myctophid Electrona carlsbergi. Hence, biochemical analysis of both WE and TAG strongly suggested that adult short-tailed shearwaters mainly prey upon Antarctic/subAntarctic myctophids when they feed for themselves, thus emphasizing the role of these oceanic mesopelagic fish in the marine ecosystem of the Southern Ocean.
\end{abstract}

KEY WORDS: Antarctica $\cdot$ Short-tailed shearwater $\cdot$ Fatty alcohols $\cdot$ Fatty acids · Trophic interactions · Myctophids · Tasmania

Resale or republication not permitted without written consent of the publisher

\section{INTRODUCTION}

In the Austral Ocean, Procellariiformes (albatrosses and petrels) dominate the seabird community in terms of specific diversity (Marchant \& Higgins 1990). They spend the majority of their life at sea, only coming on land to breed and rear a single chick (Warham 1990). With the exception of diving petrels, all procellariiform species are characterized by the presence of oil in their proventriculus (Warham 1977). The dietary origin of this stomach oil is now accepted (Lewis 1969, Cheah \& Hansen 1970a,b, Clarke \& Prince 1976, Warham et al. 1976, Place et al. 1989). It is likely produced by mechanical rupture of lipid-rich food organisms (crustacean, fish and squid) followed by differential digestion of proteins and lipids (Clarke 1989). By concentrating dietary lipids in their stomach, adult petrels and albatrosses reduce the mass and frequency of meal 
delivery to their chicks, thus decreasing the time and energy costs involved in transporting food from patchily distributed pelagic food sources. Stomach oil also plays other significant roles, such as an energy reserve for adult birds and as an offensive/defensive weapon (Warham et al. 1976, Warham 1977).

During the breeding season, some Procellariiformes use a 2-fold foraging strategy to feed their chick: they alternately perform short trips (ST) where adults forage near the colony, and long trips (LT) where they reach more distant feeding areas (Weimerskirch et al. 1994). Parent birds gain mass during LT and lose mass during ST, indicating that LT are profitable for adults probably through a build up of energy reserves and ST are profitable for chicks through an increase in their feeding frequency. A recent energetic study showed that adult self-feeding during LT is crucial for the success of ST because most of the energy used during ST is likely to derive from the energy stored during LT (Weimerskirch et al. 2003). However, the self-feeding behaviour of adult seabirds during ST cannot be investigated through the direct method of food analysis because the available information is restricted to their stomach contents that correspond to the chick- not the adult-diet. Consequently, the trophic relationships of oceanic seabirds when they feed for themselves are essentially unknown.

Unlike stomach contents collected after ST, LT-food samples contain significant amounts of stomach oil (Weimerskirch \& Cherel 1998, Weimerskirch et al. 1999, Cherel et al. 2002), whose biochemical composition could include the lipid signature of the prey caught far away the breeding colony. Studies on fatty acid and fatty alcohol signatures to investigate trophic interactions have been proposed for many levels of the oceanic food web, ranging from the initial planktonic stages to the higher predators such as birds and marine mammals (e.g. Sargent \& Falk-Petersen 1981, Iverson 1993, Raclot et al. 1998, Best et al. 2003). Lipids in marine organisms are characterized by their great diversity and high levels of long chain polyunsaturated fatty acids (PUFA), which originate from the various primary producers: phytoplankton and seaweeds (Ackman 1980). Unlike other nutrients, dietary fatty acids of carbon chain length $>14$ can be deposited in animal tissue with little or no modification (Ackman et al. 1971). Since stomach oil is derived from mechanical rupture of fat-rich prey items, its biochemical composition should be little affected by digestive processes, and it should be therefore possible to consider the patterns of fatty alcohols and fatty acids as linked to the lipid signature of the prey ingested.

The food and feeding ecology of the short-tailed shearwater Puffinus tenuirostris and its dual foraging strategy during chick rearing were recently described (Weimerskirch \& Cherel 1998, Klomp \& Schultz 2000, Schultz \& Klomp 2000). Birds alternate an average of 2 ST lasting 1 to $4 \mathrm{~d}$ before departing for a LT lasting 8 to $19 \mathrm{~d}$. They forage from Australian to Antarctic oceanic waters during LT and remain over neritic waters close to their breeding colony during ST. While suggesting birds fed in cold waters during LT, the afore mentioned studies give no indication on the prey ingested when adult birds are self-feeding at that time. This is a crucial issue first to fully understand the adult foraging strategy, and second to determine the key species of the pelagic ecosystem because the huge population of short-tailed shearwaters (about 23 million breeding birds; Skira et al. 1985) is likely to have significant interactions in food webs.

Using the Tasmanian short-tailed shearwater as a model, the aims of this investigation were (1) to study the intra-specific variability of stomach oil composition, and (2) to test the use of the lipid-signature of stomach oil to elucidate trophic relationships of procellariiform seabirds when they feed far away their breeding grounds.

\section{MATERIALS AND METHODS}

Field study and sample collection. Field work was carried out during austral summer 1997 (between 1 and 27 March) at The Neck Game Reserve (43 $18^{\prime} \mathrm{S}$, $\left.147^{\circ} 18^{\prime} \mathrm{E}\right)$, Bruny Island, located in the south-eastern of Tasmania (Fig. 1). The methodology used is detailed in Weimerskirch \& Cherel (1998). Briefly, sticks were placed at the mouth of the burrows, so that a visit by breeding Puffinus tenuirostris could be detected by displacement of the sticks. Burrows were inspected every half-hour from dusk to midnight, and $1 \mathrm{~h}$ before dawn. The duration of individual foraging trips was defined as the time elapsed between 2 successive recoveries of the same bird. Food samples were obtained either by the 'water off-loading technique' (Wilson 1984) or through spontaneous regurgitation of adult short-tailed shearwaters on arrival back at the colony after a foraging trip, before they fed their chick. Fourteen stomach contents were collected after LT lasting from 9 to $17 \mathrm{~d}$. Diet samples were frozen and returned within $2 \mathrm{wk}$ to the laboratory in France (Centre d'Etudes Biologiques de Chizé) for subsequent analyses. Stomach contents were composed of a liquid fraction made of oil and water, and a solid fraction made of more or less digested prey. In the laboratory, each sample was thawed and drained by gravity overnight to separate the liquid fraction from the solid items. These 2 fractions were then measured in a graduated tube. 


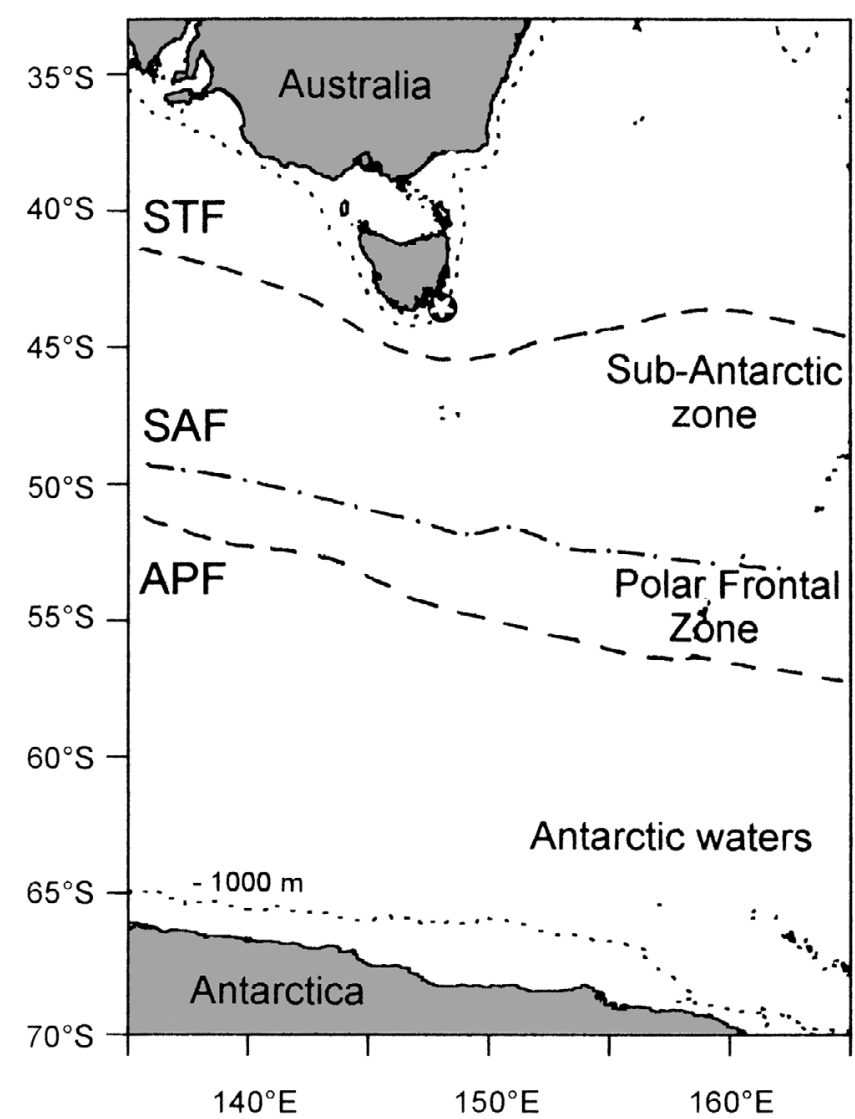

Fig. 1. Location of Bruny Island (star) off south east Tasmania in the Southern Ocean. STF: sub-tropical front; SAF: subantarctic front; APF: antarctic polar front

Prey analysis of the 14 solid fractions, i.e. identification and counting of fresh and accumulated items found in stomach contents, has been reported earlier by Weimerskirch \& Cherel (1998).

Lipid analysis. Total lipids were quantitatively extracted from each of the 14 liquid fractions according to the method of Bligh \& Dyer (1959). Crude extracts were placed in chloroform, concentrated under vacuum and stored at $-80^{\circ} \mathrm{C}$. To avoid lipid auto-oxidation, a nitrogen atmosphere was maintained at all times.

Lipid classes: Individual lipid classes were quantified using an Iatroscan MK V TH10 thin-layer chromatography-flame-ionization detector analyser (TLCFID; Iatron Laboratories; Ackman 1981). Aliquots of total extracts were applied to chromarods SIII using microcapillaries $(1 \mu \mathrm{l})$ and analysed in duplicate. Neutral lipids were separated using a double development procedure with the following solvent systems: nhexane: benzene: formic acid 80:20:1 (v/v/v) followed by $n$-hexane: diethyl ether: formic acid 97:3:1.5 (v/v/v). After development, the chromarods were oven dried and analysed immediately to minimise adsorption of atmospheric contaminants. Calibration of the rods was achieved using analytical grade commercial standards (Sigma - cholesterol [Chol], diacylglycerols (DG), free fatty acids (FFA), polar lipids (PL), triacylglycerols [TAG] and sterol esters). Peaks were quantified using ChromStar software version 4.14. Iatroscan results are generally reproducible to $\pm 10 \%$ of individual component abundance (Volkman \& Nichols 1991).

Fatty acids and fatty alcohols: Lipid classes were further isolated by preparative TLC using n-hexane: diethyl ether: acetic acid (85:15:1 v/v/v). After development, the plates were dried and sprayed with a solution of $0.2 \% 2,7$-dichlorofluorescein in ethanol. The lipids were visualised under UV light and identified by comparisons with co-chromatographed standard mixtures (Sigma). The bands of wax esters (WE) and TAG were scraped off and eluted with diethyl ether: chloroform $(1: 1 \mathrm{v} / \mathrm{v})$.

Before gas-liquid chromatography (GLC), fatty acids from both WE and TAG, and fatty alcohols from WE were converted into methyl esters and acetates, respectively. Fatty acid methyl esters were prepared using $7 \%$ boron trifluoride in methanol (Morrison \& Smith 1964). Fatty alcohols were acetylated using acetic anhydride (Ackman et al. 1972). GLC analyses were performed with an Autosystem XL gas chromatograph (Perkin Elmer) equipped with a polar column Famewax (Restek), $30 \mathrm{~m} \times 0.32 \mathrm{~mm}$ internal diameter) and a FID detector. Helium was used as carrier gas at 7 psig. The column was operated isothermally at $190^{\circ} \mathrm{C}$ during $120 \mathrm{~min}$ for fatty acid methyl esters and $200^{\circ} \mathrm{C}$ during $100 \mathrm{~min}$ for fatty alcohol acetates. Injector and detector were maintained at 225 and $250^{\circ} \mathrm{C}$, respectively. Peaks were quantified with Turbochrom Navigator software version 4.1. Individual components were tentatively identified by reference to authentic standards and well-characterized fish oils (Capelin: Menhaden 1:1). In addition to the examination of esters and acetates recovered, 1 part of fatty acid methyl ester or fatty alcohol acetate samples was completely hydrogenated and the products examined qualitatively and quantitatively by GLC. Corrections were made on the results according to the differential FID response to fatty acids or fatty alcohols depending on their chain-length.

Statistical analyses. The intra-specific variability of stomach oil was evaluated by cluster analyses using Euclidean distance and average linkage on lipid class composition, fatty acid and fatty alcohol profiles using Systat 9.0.

To achieve meaningful comparisons between fatty alcohol and fatty acid patterns from potential prey species and stomach oils, 3 databases were built using the majority of published studies on Tasmanian, subAntarctic and Antarctic potential prey, i.e. zooplankton, micronekton and nekton (Table 1). The first database 
regrouped 86 fatty alcohol profiles of 10 species of crustacean and fish (database WEAL). The second one regrouped the WE fatty acid profiles (database WEAC), and the third one regrouped the TAG fatty acid profiles of potential prey (database TAG). Unfortunately, most of studies about sub-Antarctic and Antarctic species only presented the fatty acid composition of total lipids. In this case, we assigned the fatty acid data to either the second or the third database, based on the percent composition of the prey lipids (more than $60 \%$ of WE in database WEAC and more than $60 \%$ of TAG in database TAG). Hence, in the second database, 64 profiles belonging to 9 species of crustacean and fish were compiled, while in the third one 71 profiles belonging to 19 species of crustacean, fish and squid were grouped. The data in each database were validated by performing a preliminary principal component analysis to check the homogeneity of the fatty alcohol and fatty acid patterns within a given species or group of species. The outliers were removed from the groupings used in the further discriminant analyses. Similarly, when the literature indicated conflicting results that could not be easily resolved, the corresponding species was not considered in the analyses.

Linear and Stepwise linear discriminant analyses (LDA and SLDA) were used to classify prey species based on either fatty alcohol or fatty acid patterns. The number of descriptors considered in the analyses was limited to the data available from the literature. For the WEAL and WEAC databases, LDA were performed using 8 fatty alcohols and 17 fatty acids, respectively. Then, stomach oil samples were attributed to preexisting prey group. Stomach oils were used as supplementary observations and were not used in the definition of the discriminant functions. For the TAG database, fatty acid data were analysed using the same

Table 1. Prey species gathered from the literature and included in the 3 databases. WEAL: fatty alcohol profiles of wax esters; WEAC: fatty acid profiles of wax esters; TAG: fatty acid profiles of triacylglycerols; Code: acronyms used in the statistical analyses

\begin{tabular}{|c|c|c|c|c|c|}
\hline Species & Code & WEAL & $\begin{array}{l}\text { atabase } \\
\text { WEAC }\end{array}$ & TAG & Source \\
\hline \multicolumn{6}{|l|}{ Crustacean } \\
\hline Calanoides acutus & Caа & $\mathrm{X}$ & $\mathrm{X}$ & & $\begin{array}{l}\text { Albers et al. (1996), Falk-Petersen et al. (1999), } \\
\text { Hagen et al. (1993), Kattner et al. (1994) }\end{array}$ \\
\hline Calanus propinquus & Cap & & & $\mathrm{X}$ & $\begin{array}{l}\text { Albers et al. (1996), Falk-Petersen et al. (1999), } \\
\text { Hagen et al. (1993), Kattner et al. (1994) }\end{array}$ \\
\hline Euchirella rostromagna & Eur & $\mathrm{X}$ & $\mathrm{X}$ & $\mathrm{X}$ & Albers et al. (1996), Hagen et al. (1995) \\
\hline Euphausia crystallorophias & Euc & $\mathrm{X}$ & $\mathrm{X}$ & $\mathrm{X}$ & $\begin{array}{l}\text { Bottino (1975), Falk-Petersen et al. (1999), } \\
\text { Kattner \& Hagen (1998) }\end{array}$ \\
\hline Euphausia superba & Eus & & & $\mathrm{X}$ & Mayzaud (pers. comm.) \\
\hline Euphausia vallentini & Euv & & & $\mathrm{X}$ & Mayzaud et al. (2003) \\
\hline Paraeuchaeta antarctica & Paa & $\mathrm{X}$ & $\mathrm{X}$ & & $\begin{array}{l}\text { Albers et al. (1996), Hagen et al. (1995), Mayzaud } \\
\text { (pers. comm.) }\end{array}$ \\
\hline Rhincalanus gigas & Rhg & $\mathrm{X}$ & $\mathrm{X}$ & & Kattner et al. (1994), Reinhardt \& Van Vleet (1986) \\
\hline Themisto gaudichaudii & Thg & & & $\mathrm{X}$ & $\begin{array}{l}\text { Fricke \& Oehlenschläger (1988), Nelson et al. (2001), } \\
\text { Phleger et al. (1998) }\end{array}$ \\
\hline Thysanoessa macrura & Thm & $\mathrm{X}$ & $\mathrm{X}$ & & Falk-Petersen et al. (1999), Mayzaud et al. (2003) \\
\hline \multicolumn{6}{|l|}{ Fish } \\
\hline Electrona antarctica & Ea & $\mathrm{X}$ & $\mathrm{X}$ & & Lea et al. (2002). Phleger et al. (1997) \\
\hline Electrona carlsbergi & Ec & $\mathrm{X}$ & & $\mathrm{X}$ & Lea et al. (2002), Phleger et al. (1999), Phleger et al. (1997) \\
\hline Gymnoscopelus braueri & $\mathrm{Gb}$ & $\mathrm{X}$ & $\mathrm{X}$ & & Phleger et al. (1999) \\
\hline Gymnoscopelus fraseri & Gf & & & $\mathrm{X}$ & Lea et al. (2002) \\
\hline Gymnoscopelus nicholsi & Gn & & & $\mathrm{X}$ & Lea et al. (2002), Phleger et al. (1999) \\
\hline Gymnoscopelus opisthopterus & Go & & & $\mathrm{X}$ & Phleger et al. (1999) \\
\hline Gymnoscopelus piabilis & Gp & & & $\mathrm{X}$ & Lea et al. (2002) \\
\hline Krefftichthys anderssoni & $\mathrm{Ka}$ & $\mathrm{X}$ & $\mathrm{X}$ & & Phleger et al. (1999), Nelson (pers. comm.) \\
\hline Pagothenia borchgrevinki & $\mathrm{Pb}$ & & & $\mathrm{X}$ & Phleger et al. (1999) \\
\hline Protomyctophum tenisoni & Pt & & & $\mathrm{X}$ & Lea et al. (2002) \\
\hline Trematomus bernacchii & $\mathrm{Tb}$ & & & $\mathrm{X}$ & Phleger et al. (1999) \\
\hline Trematomus hansoni & Th & & & $\mathrm{X}$ & Phleger et al. (1999) \\
\hline Trematomus newnesi & Tn & & & $\mathrm{X}$ & Phleger et al. (1999) \\
\hline Trematomus pennellii & $\mathrm{Tp}$ & & & $\mathrm{X}$ & Phleger et al. (1999) \\
\hline \multicolumn{6}{|l|}{ Cephalopod } \\
\hline Moroteuthis ingens & $\mathrm{Mi}$ & & & $\mathrm{X}$ & Phillips et al. (2001) \\
\hline Moroteuthis robsoni & $\mathrm{Mr}$ & & & $\mathrm{X}$ & Phillips et al. (2002) \\
\hline
\end{tabular}


procedure but with SLDA to optimise classification. All discriminant analyses were conducted using Statgraphics Plus 5.0. Normality being presupposed for most of these analyses, percentages were normalized using the arcsine transformation (Zar 1984).

\section{RESULTS}

Stomach contents collected after a LT showed accumulated solid components and variable amounts of orange-coloured oil (mean value $14.6 \pm 12.7 \mathrm{ml}$, range: 3.5 to $55.0 \mathrm{ml}$ ). This oily fraction represented $44 \%$ of the total volume of the liquid fraction, the remaining being water.

\section{Lipid composition}

The 14 stomach oils were mostly composed of WE and TAG. Other lipid classes (Chol, PL, FFA and DG) were present, but in small amounts (Table 2). Nine oils were WE-rich (76 to $86 \%$ of total lipids), whereas 5 oils were characterized by a mean composition in WE and TAG (49 to $65 \%$ and 28 to $41 \%$ of total lipids, respectively).

\section{Fatty acid and fatty alcohol profiles}

\section{Triacylglycerols}

Twenty-one different fatty acids were found at levels exceeding traces $(>0.5 \%$ ) representing 90 to $94 \%$ of the total fatty acids (Table 3). Monounsaturated fatty acids (MUFA; 46 to $56 \%$ ) were more prevalent than both saturated fatty acids (SFA; 22 to $31 \%$ ) and PUFA (11 to $20 \%$ ). Two fatty acids dominated by mass (>10\%): the oleic (18:1n-9; 19 to $28 \%)$ and palmitic acids (16:0; 14 to $17 \%$ ). Main fatty acids (>5\%) included the palmitoleic acid $(16: 1 \mathrm{n}-7 ; 6$ to $10 \%)$, the myristic acid $(14: 0 ; 3$ to $11 \%$ ), the eicosapentaenoic acid (EPA, 20:5n-3; 3 to $8 \%$ ), the docosahexaenoic acid (DHA, 22:6n-3; 4 to $8 \%$ ) and the $18: 1 \mathrm{n}-7$ ( 4 to $8 \%$ ) in a decreasing order of importance (Table 3). Together, these 7 fatty acids accounted for 63 to $79 \%$ of the total fatty acids. PUFA of the $n-3$ series were 3 to 6 times more abundant (10 to 19 versus $3 \%$ ) than those of the $n-6$ series.

Two main groups of oils could be discriminated on the basis of their percentages in 22:1, 24:1 and EPA/DHA ratio. This partition of oils, confirmed by cluster analysis (Fig. 2), resembles that previously observed in lipid class composition (see above).

\section{Wax esters}

Twenty-two different fatty acids were present at levels $>0.5 \%$ in all stomach oils (Table 4 ). The MUFA clearly dominated the profiles (66 to $79 \%$ of the total fatty acids). The major fatty acid was 18:1n-9 (36 to $43 \%$ ) and there were substantial amounts of 16:1n-7 (13 to $16 \%$ ), 20:5n-3 (5 to $12 \%$ ) and $22: 6 n-3$ (4 to $8 \%$ ). PUFA of the $n-3$ series were 3 to 7 more abundant than those of the n- 6 series.

Eighteen different fatty alcohols were identified $(>0.5 \%$, Table 5). More than half of components were saturated (52 to $62 \%$ ), while monounsaturated fatty alcohols represented 34 to $45 \%$ of the total. Very few polyunsaturated components were identified. The principal fatty alcohols included palmitol $(16: 0 ; 38$ to $47 \%)$, gadoleol (20:1n-9; 8 to $12 \%), 14: 0$ (7 to $9 \%$ ) and $22: 1 n-13+11$ ( 5 to $9 \%$ ).

\section{Dietary implications of stomach oil analyses}

Fatty alcohol descriptors of wax esters

To investigate species variation in the potential prey database, we conducted a LDA of the 9 major groups of prey species. LDA was performed using the 8 major fatty alcohol descriptors. Classes have been defined for each prey species except for the 2 myctophid fish Gymnoscopelus braueri and Krefftichthys anderssoni,

Table 2. Puffinus tenuirostris. Lipid composition (\% of total lipids) of the 14 stomach oils. WE: wax esters; TAG: triacylglycerols; FFA: free fatty acids; Chol: cholesterol; DG: diacylglycerols; PL: polar lipids; -: not detected

\begin{tabular}{|lccccccccccccccc|}
\hline & 1 & 2 & 3 & 4 & 5 & 6 & 7 & 8 & 9 & 10 & 11 & 12 & 13 & 14 & Mean \pm SD \\
\hline WE & 75.7 & 84.1 & 51.7 & 65.0 & 80.5 & 76.1 & 84.3 & 54.7 & 48.9 & 81.6 & 83.2 & 58.4 & 82.8 & 85.6 & $72.3 \pm 13.6$ \\
TAG & 9.0 & 7.0 & 38.4 & 28.3 & 13.9 & 18.8 & 10.9 & 38.7 & 40.9 & 13.8 & 12.3 & 31.7 & 11.5 & 10.3 & $20.4 \pm 12.4$ \\
FFA & 3.7 & 4.0 & 1.9 & 0.9 & - & - & - & 1.0 & 2.0 & - & - & 4.0 & 1.9 & 1.6 & $1.5 \pm 1.5$ \\
Chol & 9.3 & 4.2 & 5.6 & 5.0 & 4.4 & 3.9 & 4.0 & 3.2 & 4.8 & 3.2 & 2.9 & 4.7 & 2.3 & 1.1 & $4.2 \pm 1.9$ \\
DG & 0.7 & - & - & - & 0.4 & 0.5 & 0.2 & 1.1 & 1.7 & 0.4 & - & - & 0.5 & - & $0.4 \pm 0.5$ \\
PL & 1.6 & 0.7 & 2.5 & 0.8 & 0.8 & 0.7 & 0.6 & 1.2 & 1.7 & 1.0 & 1.7 & 1.1 & 1.0 & 1.3 & $1.2 \pm 0.5$ \\
a Could include other sterols & & & & & & & & & & & & \\
\hline
\end{tabular}


Table 3. Puffinus tenuirostris. Triacylglycerol fatty acid compositions (\% of triacylglycerol fatty acids) of the 14 stomach oils SD: standard deviation; SFA: saturated fatty acids; MUFA: monounsaturated fatty acids; PUFA: polyunsaturated fatty acids; EPA: 20:5n-3; DHA: 22:6n-3; -: not detected; 'Others' includes all fatty acids present at $<0.5 \%$

\begin{tabular}{|c|c|c|c|c|c|c|c|c|c|c|c|c|c|c|c|}
\hline & 1 & 2 & 3 & 4 & 5 & 6 & 7 & 8 & 9 & 10 & 11 & 12 & 13 & 14 & Mean $\pm \mathrm{SD}$ \\
\hline $14: 0$ & 6.6 & 4.1 & 10.6 & 9.9 & 3.7 & 7.7 & 4.4 & 11.0 & 9.5 & 3.0 & 4.8 & 11.4 & 5.5 & 4.1 & $6.9 \pm 3.0$ \\
\hline $16: 0$ & 16.6 & 16.8 & 14.7 & 15.5 & 16.4 & 15.5 & 15.2 & 16.6 & 14.3 & 16.8 & 17.5 & 16.2 & 15.4 & 15.7 & $15.9 \pm 0.9$ \\
\hline $18: 0$ & 1.7 & 1.9 & 2.2 & 2.6 & 1.5 & 2.0 & 1.4 & 1.8 & 2.3 & 2.8 & 2.0 & 2.4 & 1.9 & 1.8 & $2.0 \pm 0.4$ \\
\hline Phytanate & 0.6 & 1.1 & 0.1 & - & 0.6 & 0.4 & 0.6 & 0.4 & 0.4 & 0.6 & 0.9 & 0.5 & 0.6 & 0.6 & $0.6 \pm 0.2$ \\
\hline Total SFA & 25.5 & 23.9 & 27.5 & 28.0 & 22.3 & 25.6 & 21.7 & 29.8 & 26.5 & 23.2 & 25.2 & 30.5 & 23.3 & 22.2 & $25.4 \pm 2.8$ \\
\hline $16: 1 \mathrm{n}-7$ & 10.0 & 8.2 & 9.5 & 9.8 & 10.1 & 10.1 & 10.3 & 10.2 & 9.4 & 6.4 & 9.0 & 9.9 & 10.1 & 9.3 & $9.4 \pm 1.1$ \\
\hline $18: 1 n-9$ & 22.2 & 22.3 & 26.0 & 25.5 & 21.4 & 23.0 & 19.1 & 21.2 & 27.8 & 21.3 & 23.8 & 27.2 & 22.3 & 21.9 & $23.2 \pm 2.5$ \\
\hline $18: 1 \mathrm{n}-7$ & 5.5 & 4.4 & 7.6 & 7.4 & 3.8 & 5.6 & 3.8 & 6.6 & 7.7 & 3.7 & 4.2 & 7.1 & 5.1 & 4.2 & $5.5 \pm 1.5$ \\
\hline $18: 1 n-5$ & 0.7 & 0.6 & 0.4 & 0.4 & 0.8 & 0.6 & 0.7 & 0.6 & 0.4 & 0.7 & 0.7 & 0.4 & 0.7 & 0.8 & $0.6 \pm 0.2$ \\
\hline $20: 1 n-9$ & 2.4 & 2.6 & 3.0 & 2.2 & 2.4 & 2.7 & 1.9 & 2.1 & 3.6 & 6.0 & 2.1 & 2.9 & 1.9 & 2.5 & $2.7 \pm 1.0$ \\
\hline $22: 1 n-13+11$ & 3.4 & 7.7 & 0.7 & 1.1 & 7.1 & 3.5 & 5.8 & 2.1 & 1.4 & 6.8 & 6.1 & 0.9 & 5.0 & 7.1 & $4.2 \pm 2.6$ \\
\hline $22: 1 n-9$ & 1.7 & 3.5 & 0.9 & 1.2 & 3.0 & 2.0 & 2.4 & 1.9 & 1.5 & 2.4 & 2.4 & 1.1 & 2.5 & 2.9 & $2.1 \pm 0.8$ \\
\hline $24: 1 n-13$ & 0.4 & 0.8 & - & - & 0.7 & 0.3 & 0.7 & 0.2 & 0.2 & 0.6 & 0.7 & 0.1 & 0.4 & 0.6 & $0.5 \pm 0.2$ \\
\hline $24: 1 n-11$ & 1.3 & 3.4 & 0.2 & 0.8 & 3.0 & 1.4 & 2.3 & 0.6 & 0.5 & 1.9 & 2.6 & 0.2 & 2.1 & 3.0 & $1.7 \pm 1.1$ \\
\hline $24: 1 \mathrm{n}-9$ & 1.4 & 2.8 & 0.5 & 0.8 & 2.3 & 1.2 & 2.2 & 0.9 & 1.1 & 2.3 & 2.3 & 0.6 & 1.8 & 2.1 & $1.6 \pm 0.8$ \\
\hline Total MUFA & 49.1 & 56.1 & 48.7 & 49.1 & 54.6 & 50.5 & 49.1 & 46.4 & 53.5 & 51.9 & 53.9 & 50.5 & 51.9 & 54.4 & $51.4 \pm 2.8$ \\
\hline $18: 2 n-6$ & 1.5 & 1.0 & 1.8 & 1.9 & 1.1 & 1.5 & 1.4 & 1.7 & 1.7 & 1.2 & 1.2 & 1.5 & 1.5 & 1.3 & $1.4 \pm 0.3$ \\
\hline $16: 3 n-6$ & 0.5 & 0.6 & 0.5 & 0.9 & 0.7 & 0.4 & 0.7 & 0.2 & 0.4 & 0.8 & 0.6 & 0.2 & 0.6 & 0.8 & $0.6 \pm 0.2$ \\
\hline $18: 4 n-3$ & 1.7 & 0.6 & 1.2 & 1.2 & 1.6 & 1.6 & 2.1 & 1.4 & 0.9 & 0.8 & 1.0 & 0.8 & 1.5 & 1.1 & $1.2 \pm 0.4$ \\
\hline $20: 4 n-3$ & 0.6 & 0.3 & 0.7 & 0.4 & 0.5 & 0.6 & 0.6 & 0.5 & 0.5 & 0.5 & 0.4 & 0.5 & 0.6 & 0.5 & $0.5 \pm 0.1$ \\
\hline $20: 5 n-3$ & 6.6 & 2.6 & 8.3 & 5.8 & 4.7 & 6.5 & 6.6 & 7.7 & 6.0 & 3.9 & 3.6 & 6.0 & 5.7 & 4.5 & $5.6 \pm 1.6$ \\
\hline $22: 5 n-3$ & 0.5 & 0.4 & 0.6 & 0.4 & 0.5 & 0.5 & 0.6 & 0.5 & 0.5 & 0.7 & 0.4 & 0.5 & 0.5 & 0.5 & $0.5 \pm 0.1$ \\
\hline $22: 6 n-3$ & 6.4 & 5.2 & 4.8 & 3.8 & 5.8 & 5.7 & 7.7 & 4.5 & 4.2 & 7.7 & 5.7 & 4.2 & 6.2 & 6.8 & $5.6 \pm 1.3$ \\
\hline Total PUFA & 17.8 & 10.7 & 17.7 & 14.4 & 14.9 & 16.8 & 19.7 & 16.4 & 14.2 & 15.6 & 12.9 & 13.6 & 16.5 & 15.5 & $15.5 \pm 2.3$ \\
\hline Others & 7.6 & 9.4 & 6.0 & 8.6 & 8.2 & 7.1 & 9.6 & 7.4 & 5.7 & 9.2 & 8.0 & 5.5 & 8.2 & 8.0 & $7.8 \pm 1.3$ \\
\hline Total n-3 & 16.7 & 9.6 & 16.4 & 12.2 & 14.0 & 15.7 & 18.8 & 15.4 & 12.8 & 14.4 & 11.7 & 12.5 & 15.3 & 14.3 & $14.3 \pm 2.4$ \\
\hline Total n-6 & 3.0 & 2.4 & 3.4 & 3.7 & 2.8 & 2.9 & 3.3 & 2.9 & 2.9 & 3.2 & 2.5 & 2.6 & 3.1 & 2.9 & $3.0 \pm 0.3$ \\
\hline Ratio n-3/n-6 & 5.6 & 4.0 & 4.9 & 3.3 & 5.0 & 5.4 & 5.7 & 5.3 & 4.4 & 4.6 & 4.6 & 4.7 & 5.0 & 4.9 & $4.8 \pm 0.6$ \\
\hline EPA/DHA & 1.0 & 0.5 & 1.7 & 1.5 & 0.8 & 1.1 & 0.8 & 1.7 & 1.4 & 0.5 & 0.6 & 1.4 & 0.9 & 0.7 & $1.1 \pm 0.4$ \\
\hline
\end{tabular}

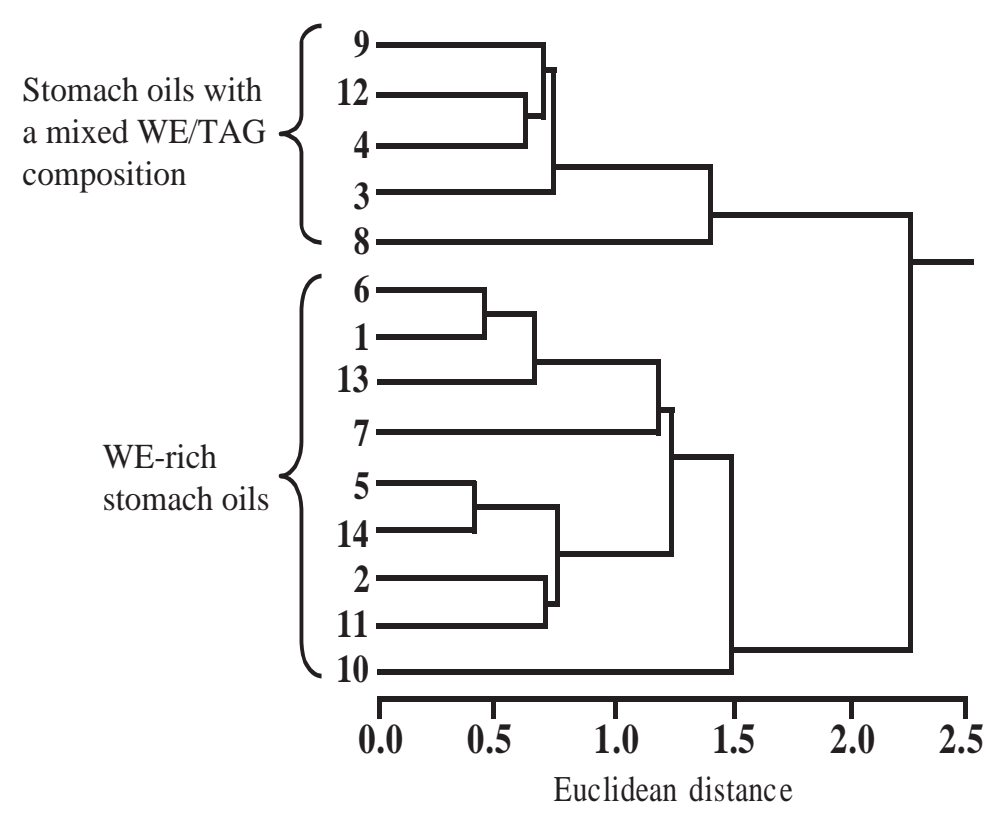

Fig. 2. Puffinus tenuirostris. Classification by clustering of the 14 stomach oils (1 to 14 ) based on the fatty acid profiles of triacylglycerols (TAG). WE: wax esters which were regrouped in a single set (Gb/Ka). Four discriminant functions accounted for $99 \%$ of the total inertia. A plot of the scores of the first 2 discriminant functions (representing $88 \%$ of the variance) showed single species groups (Calanoides acutus [Caa], Thysanoessa macrura [Thm], Electrona carlsbergi [Ec]) and 2 groups of species with relatively similar fatty alcohol profiles, i.e. the fish species Electrona antarctica (Ea) together with the fish group $\mathrm{Gb} / \mathrm{Ka}$, and the copepodeuphausiid group: Rhincalanus gigas (Rhg), Euphausia crystallorophias (Euc), Paraeuchaeta antarctica (Paa) and Euchirella rostromagna (Eur) (Fig. 3A). The first function, which explained $73 \%$ of the inertia, is linked to 2 groups, the fish species Ec and the euphausiid Thm in opposition to other zooplankton species Euc, Eur, Paa, and Rhg. This function contrasted between species that showed very low levels of 14:0 and high percentages of 18:1n-9, and other zooplankton species having significantly higher levels of 14:0. The second function, which accounted for $14.5 \%$ of the inertia, was related to Ec, lacking 20:1 alcohol, in opposition to the copepod Caa. Using this model of discriminant functions, $100 \%$ of the cases were correctly assigned. 
Table 4. Puffinus tenuirostris. Wax ester fatty acid compositions (\% of wax ester fatty acids) of the 14 stomach oils. SD: standard deviation; SFA: saturated fatty acids; MUFA: monounsaturated fatty acids; PUFA: polyunsaturated fatty acids; EPA: 20:5n-3; DHA: 22:6n-3; 'Others' includes all fatty acids present at $<0.5 \%$

\begin{tabular}{|c|c|c|c|c|c|c|c|c|c|c|c|c|c|c|c|}
\hline & 1 & 2 & 3 & 4 & 5 & 6 & 7 & 8 & 9 & 10 & 11 & 12 & 13 & 14 & Mean \pm SD \\
\hline $14: 0$ & 1.6 & 0.8 & 1.7 & 1.6 & 0.8 & 1.1 & 0.8 & 0.8 & 1.8 & 0.7 & 0.6 & 1.7 & 0.9 & 0.9 & $1.1 \pm 0.4$ \\
\hline $16: 0$ & 1.1 & 0.9 & 1.2 & 1.4 & 0.7 & 0.9 & 0.9 & 0.9 & 1.8 & 0.8 & 0.7 & 1.5 & 0.9 & 0.8 & $1.0 \pm 0.3$ \\
\hline Total SFA & 2.6 & 1.7 & 2.9 & 3.0 & 1.5 & 2.0 & 1.7 & 1.7 & 3.6 & 1.5 & 1.4 & 3.2 & 1.7 & 1.6 & $2.1 \pm 0.7$ \\
\hline $16: 1 \mathrm{n}-7$ & 14.2 & 13.7 & 15.2 & 13.8 & 14.9 & 15.4 & 14.9 & 14.0 & 14.8 & 14.1 & 12.6 & 13.6 & 14.5 & 16.3 & $14.4 \pm 0.9$ \\
\hline $17: 1$ & 0.8 & 0.8 & 0.7 & 0.7 & 0.8 & 0.8 & 0.7 & 0.8 & 0.8 & 0.8 & 0.7 & 0.7 & 0.8 & 0.8 & $0.8 \pm 0.1$ \\
\hline $18: 1 \mathrm{n}-9$ & 40.0 & 37.8 & 39.9 & 38.6 & 40.2 & 39.3 & 39.2 & 38.3 & 43.0 & 38.3 & 35.7 & 39.9 & 38.6 & 42.0 & $39.3 \pm 1.8$ \\
\hline $18: 1 \mathrm{n}-7$ & 4.5 & 3.8 & 5.0 & 4.3 & 4.3 & 4.4 & 4.1 & 3.9 & 4.6 & 3.8 & 3.4 & 5.7 & 4.0 & 4.0 & $4.3 \pm 0.6$ \\
\hline $18: 1 \mathrm{n}-5$ & 0.7 & 0.8 & 0.8 & 0.6 & 0.8 & 0.8 & 0.7 & 0.7 & 0.8 & 0.7 & 0.8 & 0.7 & 0.8 & 0.6 & $0.7 \pm 0.1$ \\
\hline $20: 1 n-11$ & 0.7 & 0.7 & 0.7 & 0.6 & 0.9 & 0.8 & 0.9 & 0.7 & 0.7 & 0.7 & 0.7 & 0.4 & 0.8 & 1.1 & $0.7 \pm 0.2$ \\
\hline $20: 1 n-9$ & 4.0 & 4.0 & 4.2 & 3.7 & 4.3 & 4.2 & 4.4 & 3.1 & 3.4 & 4.6 & 4.4 & 4.2 & 3.8 & 4.6 & $4.1 \pm 0.4$ \\
\hline 22:1n-13+11 & 3.7 & 5.5 & 3.5 & 3.5 & 5.8 & 5.4 & 5.7 & 3.8 & 3.9 & 4.6 & 4.6 & 2.6 & 5.0 & 6.5 & $4.6 \pm 1.1$ \\
\hline $22: 1 n-9$ & 1.1 & 1.8 & 1.1 & 1.0 & 1.8 & 1.6 & 1.7 & 1.2 & 1.3 & 1.2 & 1.5 & 1.0 & 1.6 & 1.8 & $1.4 \pm 0.3$ \\
\hline $24: 1 n-13$ & 0.5 & 0.7 & 0.4 & 0.5 & 0.9 & 0.7 & 0.7 & 0.5 & 0.4 & 0.6 & 0.6 & 0.2 & 0.7 & 0.7 & $0.6 \pm 0.2$ \\
\hline 24:1n-11 & 0.5 & 1.1 & 0.4 & 0.4 & 1.0 & 0.9 & 0.9 & 0.6 & 0.7 & 0.7 & 0.7 & 0.2 & 0.8 & 1.0 & $0.7 \pm 0.3$ \\
\hline Total MUFA & 70.6 & 70.7 & 71.8 & 67.8 & 75.7 & 74.2 & 74.0 & 67.4 & 74.3 & 70.0 & 65.8 & 69.4 & 71.4 & 79.4 & $71.6 \pm 3.6$ \\
\hline $18: 2 n-6$ & 2.1 & 1.7 & 2.1 & 2.1 & 1.8 & 1.8 & 1.7 & 2.0 & 2.1 & 1.9 & 1.8 & 2.1 & 1.9 & 1.8 & $1.9 \pm 0.2$ \\
\hline $16: 3 n-6$ & 0.7 & 0.6 & 0.6 & 0.6 & 0.6 & 0.6 & 0.7 & 0.6 & 0.7 & 0.7 & 0.5 & 0.6 & 0.6 & 0.7 & $0.6 \pm 0.1$ \\
\hline $18: 4 n-3$ & 2.0 & 1.9 & 1.7 & 1.8 & 1.4 & 1.6 & 2.0 & 2.4 & 1.1 & 2.4 & 2.5 & 1.8 & 2.0 & 0.9 & $1.8 \pm 0.5$ \\
\hline $20: 4 n-6$ & 0.5 & 0.4 & 0.5 & 0.6 & 0.4 & 0.5 & 0.4 & 0.6 & 0.7 & 0.4 & 0.5 & 0.5 & 0.5 & 0.4 & $0.5 \pm 0.1$ \\
\hline $20: 4 n-3$ & 0.7 & 0.7 & 0.7 & 0.7 & 0.6 & 0.6 & 0.6 & 0.8 & 0.7 & 0.8 & 0.8 & 0.7 & 0.8 & 0.6 & $0.7 \pm 0.1$ \\
\hline $20: 5 n-3$ & 9.3 & 9.6 & 8.6 & 9.5 & 7.3 & 8.1 & 7.9 & 10.8 & 6.7 & 9.6 & 12.2 & 9.6 & 9.2 & 5.1 & $8.8 \pm 1.8$ \\
\hline $21: 5 n-3$ & 1.1 & 0.6 & 0.5 & 0.7 & 0.4 & 0.5 & 0.6 & 0.6 & 0.4 & 0.6 & 0.7 & 0.6 & 0.6 & 0.3 & $0.6 \pm 0.2$ \\
\hline $22: 5 n-3$ & 0.6 & 0.6 & 0.5 & 0.5 & 0.5 & 0.5 & 0.6 & 0.7 & 0.4 & 0.7 & 0.7 & 0.6 & 0.6 & 0.5 & $0.6 \pm 0.1$ \\
\hline $22: 6 n-3$ & 5.3 & 6.3 & 4.6 & 5.4 & 4.8 & 4.7 & 4.9 & 6.4 & 4.4 & 5.9 & 7.6 & 5.4 & 5.8 & 3.8 & $5.4 \pm 1.0$ \\
\hline Total PUFA & 22.1 & 22.5 & 19.7 & 22.0 & 17.9 & 18.8 & 19.3 & 24.9 & 17.1 & 23.0 & 27.2 & 21.8 & 21.9 & 14.1 & $20.9 \pm 3.3$ \\
\hline Others & 4.8 & 5.1 & 5.6 & 7.3 & 4.9 & 4.5 & 5.1 & 6.0 & 5.0 & 5.5 & 5.6 & 5.6 & 5.0 & 4.8 & $5.3 \pm 0.7$ \\
\hline Total n-3 & 19.4 & 20.4 & 16.9 & 19.3 & 15.6 & 16.4 & 17.0 & 22.2 & 14.1 & 20.5 & 25.9 & 19.1 & 19.4 & 11.7 & $18.4 \pm 3.5$ \\
\hline Total n-6 & 4.1 & 3.7 & 4.4 & 4.2 & 3.7 & 3.8 & 3.7 & 4.4 & 4.5 & 4.0 & 3.6 & 4.2 & 3.9 & 3.8 & $4.0 \pm 0.3$ \\
\hline Ratio n-3/n-6 & 4.8 & 5.6 & 3.8 & 4.6 & 4.2 & 4.3 & 4.6 & 5.1 & 3.2 & 5.1 & 7.2 & 4.5 & 5.0 & 3.1 & $4.6 \pm 1.0$ \\
\hline EPA/DHA & 1.8 & 1.5 & 1.9 & 1.8 & 1.5 & 1.7 & 1.6 & 1.7 & 1.5 & 1.6 & 1.6 & 1.8 & 1.6 & 1.3 & $1.6 \pm 0.1$ \\
\hline
\end{tabular}

Table 5. Puffinus tenuirostris. Wax ester fatty alcohol compositions (\% of wax ester fatty alcohols) of the 14 stomach oils. SD: standard deviation; SFAl: saturated fatty alcohols; MUFAl: monounsaturated fatty alcohols; 'Others' includes all fatty alcohols present at $<0.5 \%$

\begin{tabular}{|lccccccccccccccc|}
\hline & 1 & 2 & 3 & 4 & 5 & 6 & 7 & 8 & 9 & 10 & 11 & 12 & 13 & 14 & Mean \pm SD \\
\hline Iso 17:0 & 0.6 & 0.7 & 0.6 & 0.8 & 0.6 & 0.6 & 0.6 & 0.8 & 0.8 & 0.8 & 0.7 & 0.7 & 0.8 & 0.8 & $0.7 \pm 0.1$ \\
Iso 18:0 & 0.6 & 0.5 & 0.5 & 0.6 & 0.5 & 0.5 & 0.5 & 0.6 & 0.5 & 0.6 & 0.5 & 0.6 & 0.6 & 0.5 & $0.5 \pm 0.0$ \\
$14: 0$ & 8.6 & 9.0 & 8.1 & 8.9 & 8.6 & 9.0 & 7.7 & 8.7 & 9.2 & 7.4 & 8.6 & 9.4 & 8.6 & 7.8 & $8.5 \pm 0.6$ \\
$15: 0$ & 0.7 & 0.8 & 0.6 & 0.7 & 0.7 & 0.7 & 0.7 & 0.7 & 0.8 & 0.6 & 0.7 & 0.6 & 0.7 & 0.7 & $0.7 \pm 0.1$ \\
$16: 0$ & 42.3 & 45.2 & 38.3 & 41.0 & 43.2 & 41.5 & 41.0 & 45.1 & 47.2 & 39.9 & 43.7 & 41.4 & 43.4 & 39.4 & $42.3 \pm 2.5$ \\
$18: 0$ & 3.0 & 3.4 & 3.3 & 2.9 & 3.4 & 3.4 & 3.3 & 3.3 & 3.5 & 3.0 & 3.3 & 3.2 & 3.3 & 3.0 & $3.2 \pm 0.2$ \\
$20: 0$ & 0.4 & 0.6 & 0.3 & 0.3 & 0.6 & 0.6 & 0.5 & 0.4 & 0.5 & 0.4 & 0.5 & 0.3 & 0.5 & 0.5 & $0.5 \pm 0.1$ \\
Total SFAl & 56.2 & 60.2 & 51.6 & 55.2 & 57.6 & 56.3 & 54.3 & 59.6 & 62.4 & 52.7 & 58.0 & 56.2 & 57.8 & 52.7 & $56.5 \pm 3.1$ \\
$16: 1 \mathrm{n}-7$ & 4.7 & 4.3 & 4.5 & 4.9 & 4.3 & 4.3 & 4.3 & 4.9 & 4.2 & 4.6 & 4.4 & 4.5 & 4.6 & 4.2 & $4.5 \pm 0.2$ \\
$18: 1 \mathrm{n}-9$ & 4.3 & 2.8 & 5.1 & 4.8 & 3.0 & 3.3 & 3.0 & 3.8 & 4.0 & 3.6 & 3.0 & 6.9 & 2.9 & 3.0 & $3.8 \pm 1.1$ \\
$18: 1 \mathrm{n}-7$ & 4.2 & 2.4 & 5.3 & 4.5 & 2.6 & 3.2 & 2.5 & 3.1 & 3.9 & 2.9 & 2.6 & 6.3 & 2.6 & 2.8 & $3.5 \pm 1.2$ \\
$18: 1 \mathrm{n}-5$ & 1.1 & 0.8 & 1.2 & 1.2 & 1.0 & 1.1 & 0.9 & 1.2 & 1.0 & 1.3 & 1.1 & 1.1 & 0.8 & 1.2 & $1.1 \pm 0.2$ \\
$20: 1 \mathrm{n}-9$ & 10.6 & 8.8 & 12.3 & 10.4 & 9.8 & 10.1 & 10.9 & 8.0 & 7.6 & 12.2 & 10.1 & 10.1 & 9.8 & 11.2 & $10.1 \pm 1.4$ \\
$20: 1 \mathrm{n}-7$ & 0.5 & 0.4 & 0.6 & 0.6 & 0.5 & 0.5 & 1.1 & 0.7 & 0.7 & 0.8 & 0.6 & 0.8 & 0.5 & 0.5 & $0.6 \pm 0.2$ \\
$22: 1 \mathrm{n}-13+11$ & 6.7 & 7.6 & 7.5 & 6.6 & 7.9 & 8.0 & 9.6 & 6.9 & 5.4 & 9.1 & 7.4 & 4.7 & 7.8 & 9.4 & $7.5 \pm 1.4$ \\
$22: 1 \mathrm{n}-9$ & 4.6 & 4.8 & 4.5 & 4.1 & 5.2 & 5.1 & 5.2 & 3.5 & 3.3 & 4.7 & 4.9 & 3.6 & 5.2 & 6.2 & $4.6 \pm 0.8$ \\
$22: 1 \mathrm{n}-7$ & 0.4 & 0.5 & 0.4 & 0.4 & 0.5 & 0.6 & 0.6 & 0.4 & 0.4 & 0.6 & 0.4 & 0.3 & 0.5 & 0.6 & $0.5 \pm 0.1$ \\
$24: 1 \mathrm{n}-11$ & 0.8 & 1.2 & 0.8 & 0.7 & 1.4 & 1.1 & 1.2 & 0.9 & 0.6 & 1.1 & 1.2 & 0.3 & 1.0 & 1.4 & $1.0 \pm 0.3$ \\
$24: 1 \mathrm{n}-9$ & 2.7 & 2.9 & 2.9 & 2.8 & 3.0 & 2.8 & 3.2 & 3.0 & 2.6 & 2.9 & 2.7 & 2.1 & 3.1 & 3.2 & $2.8 \pm 0.3$ \\
Total MUFAl & 40.5 & 36.4 & 45.0 & 40.9 & 39.1 & 40.0 & 42.4 & 36.5 & 33.6 & 43.7 & 38.3 & 40.5 & 38.7 & 43.6 & $39.9 \pm 3.2$ \\
Others & 3.4 & 3.4 & 3.3 & 3.9 & 3.4 & 3.7 & 3.3 & 3.8 & 3.9 & 3.7 & 3.8 & 3.3 & 3.5 & 3.7 & $3.6 \pm 0.2$ \\
& & & & & & & & & & & & & & \\
\hline
\end{tabular}



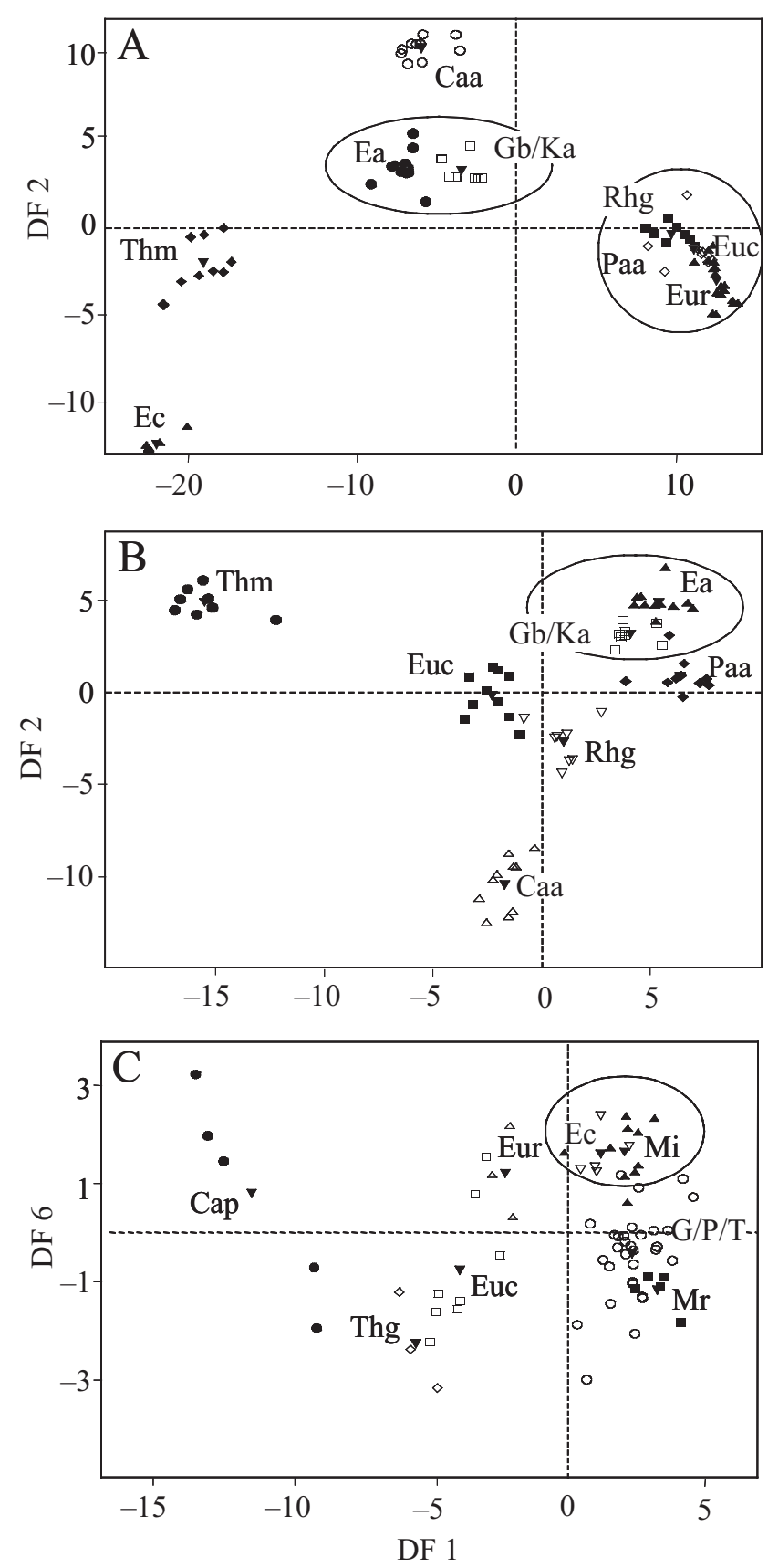

Fig. 3. Discriminant scores of (A) fatty alcohol, (B) wax ester fatty acid and (C) triacylglycerol fatty acid patterns of prey species. DF: discriminant function; see Table 1 for acronyms. G/P/T: Gymnoscopelus spp., Pagothenia borchgrevinki, Protomyctophum tenisoni, Trematomus spp.

The 14 patterns of stomach oil alcohols were then used as test samples and prediction of group allocation achieved with the same model. The results indicated highest probability of grouping with the fish group Gb/Ka. The second highest probability grouped oils with the myctophid species Ea (Table 6).
Fatty acid descriptors of wax esters and triacylglycerols

The same procedure was used with the fatty acid fraction of WE and TAG. However, the data on fatty acids from potential prey corresponded mainly to total lipids rather than specific class (WE or TAG), so the fatty acid patterns could be partially modified in relation with the proportions in the polar lipid content. Thus, these comparisons should be considered as a confirmation of the conclusion derived from the fatty alcohol patterns.

For the WE fatty acids, the score plot of the first 2 functions confirmed the grouping of Ea with Gb/Ka (Fig. 3B), a grouping still valid on the score plots with function 3 (not shown). The first 3 discriminant functions accounted for $91 \%$ of total variance. The first discriminant function represented $48 \%$ of the inertia and clearly separated the euphausiid Thm from the 8 other species (zooplankton species: Caa, Eur, Euc, Paa, Rhg; fish species: Ea, Gb and $\mathrm{Ka}$ ). This function can be interpreted as a contrast between proportions of 14:0 and 16:0 and proportion of $22: 6 n-3$. The second discriminant function accounted for $29 \%$ of the inertia. It illustrated the contrast between the proportions of 18:1n-9 and 22:6n-3, and was associated with the copepod species Caa rich in 22:6n-3 and poor in 18:1n-9, in opposition with the myctophid fish Ea rich in 18:1n-9. The third discriminant function represented $14 \%$ of the inertia and was linked with the fish species Ea. It corresponded to the presence of 24:1 associated with very low levels of 18:3n-3. With this second model of discriminant functions, $100 \%$ of the cases were correctly assigned. Based on this model, comparison of oil WE samples with the prey data showed that stomach oils presented the highest probability of association with the patterns of Ea, and Gb/Ka (Table 6) in agreement with the earlier findings.

Comparison of the TAG fatty acid patterns of stomach oils with the literature data was performed using 71 profiles from 11 fish species, 6 crustacean species and 2 cephalopod species (digestive gland data). When actual TAG profiles were not available, total fatty acid profiles were used but the very low levels of WE $(<2 \%)$ suggested that the total fatty acid patterns reflected mostly the TAG variability and to a minor extent that of the PL fraction. A SLDA was performed with the 16 major fatty acid descriptors. Classes have been defined for each prey species except for 1 set of fish species (Gymnoscopelus spp., Pagothenia borchgrevinki, Protomyctophum tenisoni, Trematomus spp.) grouped as ' $\mathrm{G} / \mathrm{P} / \mathrm{T}$ ', because of their strong similarity in fatty acid patterns. Both ascending and descending deletion resulted in a set of 10 fatty acid descriptors and 6 significant discriminant functions ( $p<0.01$ ), which accounted for $99 \%$ of the total inertia. The first 2 discriminant functions represented 46 and $28 \%$ of the 
Table 6. Discriminant analyses of prey database comparing wax ester fatty alcohol, wax ester fatty acid or triacylglycerol fatty acid signatures of prey species. WEAL: wax ester fatty alcohols; WEAC: wax ester fatty acids; TAG: triacylglycerol fatty acids; for species acronyms see Table 1. G/P/T: Gymnoscopelus spp., Pagothenia borchgrevinki, Protomyctophum tenisoni, Trematomus spp.

\begin{tabular}{|c|c|c|c|c|c|c|c|c|c|}
\hline \multirow[t]{2}{*}{ Species } & \multicolumn{3}{|c|}{ Allocated group } & \multicolumn{3}{|c|}{$\begin{array}{l}\text { First (and second) highest } \\
\text { probability group }(p<0.01)\end{array}$} & \multicolumn{3}{|c|}{ Correct classification (\%) } \\
\hline & WEAL & WEAC & TAG & WEAL & $\begin{array}{l}\text { Database } \\
\text { WEAC }\end{array}$ & TAG & WEAL & $\begin{array}{l}\text { Database } \\
\text { WEAC }\end{array}$ & TAG \\
\hline \multicolumn{10}{|l|}{ Crustacean } \\
\hline Caа & Caа & Caа & - & Caa & Caa & - & 100 & 100 & - \\
\hline Cap & - & - & Cap & - & - & Cap & - & - & 100 \\
\hline Eur & Eur & Euc & Eur & Eur & Euc & Eur & 100 & 100 & 100 \\
\hline Euc & Euc & Euc & Euc & Euc & Euc & Euc & 100 & 100 & 100 \\
\hline Eus & - & - & Euc & - & - & Euc & - & - & 100 \\
\hline Euv & - & - & Euc & - & - & Euc & - & - & 100 \\
\hline Paа & Paa & Рaа & - & Paa & Рaа & - & 100 & 100 & - \\
\hline Rhg & Rhg & Rhg & - & Rhg & Rhg & - & 100 & 100 & - \\
\hline Thg & - & - & Thg & - & - & Thg & - & - & 100 \\
\hline Thm & Thm & Thm & - & Thm & Thm & - & 100 & 100 & - \\
\hline \multicolumn{10}{|l|}{ Fish } \\
\hline $\mathrm{Ea}$ & Ea & $\mathrm{Ea}$ & - & $\mathrm{Ea}$ & $\mathrm{Ea}$ & - & 100 & 100 & - \\
\hline Ec & Ec & - & Ec & $\mathrm{Ec}$ & - & Ec & 100 & - & 100 \\
\hline $\mathrm{Gb}$ & $\mathrm{Gb} / \mathrm{Ka}$ & $\mathrm{Gb} / \mathrm{Ka}$ & - & $\mathrm{Gb} / \mathrm{Ka}$ & $\mathrm{Gb} / \mathrm{Ka}$ & - & 100 & 100 & - \\
\hline Gf & - & - & $\mathrm{G} / \mathrm{P} / \mathrm{T}$ & - & - & $\mathrm{G} / \mathrm{P} / \mathrm{T}$ & - & - & 94 \\
\hline Gn & - & - & $\mathrm{G} / \mathrm{P} / \mathrm{T}$ & - & - & $\mathrm{G} / \mathrm{P} / \mathrm{T}$ & - & - & 94 \\
\hline Go & - & - & $\mathrm{G} / \mathrm{P} / \mathrm{T}$ & - & - & $\mathrm{G} / \mathrm{P} / \mathrm{T}$ & - & - & 94 \\
\hline $\mathrm{Gp}$ & - & - & $\mathrm{G} / \mathrm{P} / \mathrm{T}$ & - & - & $\mathrm{G} / \mathrm{P} / \mathrm{T}$ & - & - & 94 \\
\hline Ка & $\mathrm{Gb} / \mathrm{Ka}$ & $\mathrm{Gb} / \mathrm{Ka}$ & - & $\mathrm{Gb} / \mathrm{Ka}$ & $\mathrm{Gb} / \mathrm{Ka}$ & - & 100 & 100 & - \\
\hline $\mathrm{Pb}$ & - & - & $\mathrm{G} / \mathrm{P} / \mathrm{T}$ & - & - & $\mathrm{G} / \mathrm{P} / \mathrm{T}$ & - & - & 94 \\
\hline $\mathrm{Pt}$ & - & - & $\mathrm{G} / \mathrm{P} / \mathrm{T}$ & - & - & $\mathrm{G} / \mathrm{P} / \mathrm{T}$ & - & - & 94 \\
\hline $\mathrm{Tb}$ & - & - & $\mathrm{G} / \mathrm{P} / \mathrm{T}$ & - & - & $\mathrm{G} / \mathrm{P} / \mathrm{T}$ & - & - & 94 \\
\hline Th & - & - & $\mathrm{G} / \mathrm{P} / \mathrm{T}$ & - & - & $\mathrm{G} / \mathrm{P} / \mathrm{T}$ & - & - & 94 \\
\hline Tn & - & - & $\mathrm{G} / \mathrm{P} / \mathrm{T}$ & - & - & $\mathrm{G} / \mathrm{P} / \mathrm{T}$ & - & - & 94 \\
\hline $\mathrm{Tp}$ & - & - & $\mathrm{G} / \mathrm{P} / \mathrm{T}$ & - & - & $\mathrm{G} / \mathrm{P} / \mathrm{T}$ & - & - & 94 \\
\hline \multicolumn{10}{|l|}{ Squid } \\
\hline Mi & - & - & Mi & - & - & Mi & - & - & 100 \\
\hline $\mathrm{Mr}$ & - & - & $\mathrm{Mr}$ & - & - & $\mathrm{Mr}$ & - & - & 100 \\
\hline Stomach oils & - & - & - & $\begin{array}{c}\mathrm{Gb} / \mathrm{Ka} \\
(\mathrm{Ea})\end{array}$ & $\begin{array}{c}\mathrm{Ea} \\
(\mathrm{Gb} / \mathrm{Ka})\end{array}$ & $\mathrm{Mi}$ & 100 & 93 & 100 \\
\hline
\end{tabular}

total variance, respectively. The first function is linked to the copepod Calanus propinquus (Cap) in relation to the changes in 16:1 and 24:1. The second function is related to an opposition between the euphausiid species Euc and the copepod Cap with variations in 24:1, 16:0 and 18:1. Of interest in the present study is the sixth function that accounted for $3 \%$ of the inertia. It contrasts the 2 cephalopod species Moroteuthis ingens and Moroteuthis robsoni ( $\mathrm{Mi}$ and $\mathrm{Mr}$, respectively) in relation with changes in 24:1 and 22:6n-3, and it singles out 1 group comprising $\mathrm{Mi}$ and Ec with very similar fatty acid patterns. This proximity of Mi and Ec fatty acid patterns suggested by the discriminant functions 1 and 6 (Fig. 3C) is confirmed by the first 2 discriminant functions. With this last model of 6 discriminant functions, $94 \%$ of the cases were correctly assigned and the prediction of stomach oil triacylglycerol allocation indicated highest probabilities of grouping with the pattern recorded in the cephalopod Mi (Table 6).

\section{DISCUSSION}

Most previous studies on procellariiform stomach oils were carried out in the 1970s. Until now, oils from 25 bird species have been analysed in terms of lipid classes, and only 12, 7 and 4 in terms of TAG fatty acid profiles, WE fatty acid and fatty alcohol profiles, respectively (Lewis 1966, 1969, Cheah \& Hansen 1970a,b, Clarke \& Prince 1976, Warham et al. 1976). Moreover, these published data are far from detailed: they presented groups of fatty acids or fatty alcohols (as 16:1, 18:2, etc.) and not the proportion of each compound. To our knowledge, the present study is the first to consider a significant number of stomach oils from adult Procellariiformes for which the duration of foraging trip was known. This greatly reduces the risk of mixing oils from different foraging trips and from different birds (males and females), as it is the case for oils from chicks. Unlike previous studies, each oil 
sample was also analysed separately to assess the intra-specific variability of lipid oil composition.

\section{Stomach oil biochemical composition}

Two lipid classes dominated in the stomach oils of Puffinus tenuirostris: WE and TAG. Our values are comparable to those reported by Woodward et al. (1995) in adult $P$. tenuirostris stomach oil and Cheah \& Hansen (1970a), Warham et al. (1976) and Bishop et al. (1983) in chick oil of the same bird species. Oils from the 25 species previously investigated also contained these main components, i.e. WE and TAG (76 and 92\% of bird species, respectively; review by Warham 1996). PL showed only very low concentrations (e.g. 0.6 to $2.5 \%$ in our study) compared with the actual percentages recorded in most of marine organisms listed in our prey species databases. This difference is probably due to the structural nature of the PL, which cannot diffuse easily following mechanical rupture and are rapidly transferred with the aqueous phase of the prey. Traces of FFA $(<4 \%)$ and absence of free fatty alcohols indicated a good degree of oil conservation.

The detailed profiles of fatty acids, from both WE and TAG and fatty alcohols, obtained in this work are consistent with earlier studies in chicks of the same bird species, where a dominance of MUFA in fatty acid profiles (WE and TAG) and SFA in fatty alcohol profiles were reported. Similarly, the major compounds recorded in our study agreed with those described in the 3 other studies (Cheah \& Hansen 1970a, Warham et al. 1976, Bishop et al. 1983). However, when considering the overall pattern of fatty acids or fatty alcohols, differences in proportions can be detected. For example, we found in our study fewer long carbon chain PUFA (20:5, 22:5 and 22:6) in TAG profiles than Cheah \& Hansen (1970a) and Warham et al. (1976) (12, 20 and 18\%, respectively).

The inter-individual degree of heterogeneity of stomach oil composition varied with the lipid class considered. Because stomach oil originated from mechanical disruption of the prey (Clarke 1989), the structure of the neutral lipids accumulated is likely to be directly linked to the oil composition of the prey ingested without the possible modifications observed when assimilation processes are involved (see Dahl et al. 2000). We have seen earlier that 2 bird groups were detected considering the lipid class composition (1 had 9 oils rich in WE and the other was composed of the remaining 5 oils with a mean composition in WE and TAG). Furthermore, if the WE were homogeneous for all 14 stomach fatty acid and fatty alcohol profiles, then the TAG structure suggested 2 groups of individuals with different fatty acid compositions corresponding to the 2 groups obtained by lipid classes. The most conser- vative hypothesis to explain this classification is that all birds would have eaten a single WE-rich prey species, and the 5 birds whose stomach oils have a mean composition WE/TAG would have preyed, in addition, on TAG-rich prey species. Indeed, as shown in the references from Table 1, most marine pelagic species displayed a single dominance of 1 neutral lipid class with either WE or TAG.

\section{Use of fatty alcohol and fatty acid patterns in trophic studies}

Different approaches using biochemical characteristics of prey have been used to describe trophic interactions. Horgan \& Barrett (1985) attempted to use lipid class composition to compare the diet of seabirds. However, 2 different prey species may present almost identical lipid class profiles due to similarities in their feeding pattern and/or life cycle. This limitation has been overcome with the use of fatty acid descriptors, as shown for myctophids by Lea et al. (2002). Specific biomarkers are in limited number and associated to the lower part of the food chain. For instance, 16:4n-1, $18: 5 n-3$ or $20: 1 n-9$ and $22: 1 n-13+11$ fatty acids are characteristics of plankton assemblages dominated by diatoms, dinoflagellates (some Haptophycae) and Calanus spp. type copepods, respectively (Ackman et al. 1964, Joseph 1975, Mayzaud et al. 1976, Pascal \& Ackman 1976). Ratios among fatty acids have also been used to clarify the dominant trophic interactions between zooplankton and phytoplankton or zooplankton and higher trophic levels. The ratios EPA/DHA, PUFA/SFA, $18: 1 n-9 / 18: 1 n-7$ or $18: 1 n-9 / 16: 1 n-7$ have often been used to discriminate between herbivores, omnivores and carnivores, for example (Cripps \& Atkinson 2000, Auel et al. 2002, Phleger et al. 2002). However, the concept of specific markers or ratios is of limited value to a study dealing with trophic interactions of higher marine predators or large-scale comparisons. Consequently, various statistical multivariate approaches have been proposed (Grahl-Nielsen \& Mjaavatten 1991, Smith et al. 1997, Mayzaud et al. 1999) to account for the covariation of entire profiles of fatty alcohols and/or fatty acids from both potential prey and predator.

Ideally, to reduce variability, the data on prey and predator should be collected simultaneously to avoid possible changes associated with season and/or location (e.g. Hagen et al. 1993, Phleger et al. 1997, Kattner \& Hagen 1998, Lea et al. 2002). Multivariate analyses of fatty acid descriptors should also be based on the same lipid classes to reduce the influence of components not directly involved in the feeding processes. In the specific case of Antarctic seabirds, the geographi- 
cal area covered during foraging trips precludes the possibility of simultaneous sampling of plankton, fish and other potential prey (mainly for logistic reasons). As a result, literature data have to be considered and sources of variability minimized as far as possible. Lipids, as trophic markers, can be used to assess diet composition in a qualitative manner. For seabirds and their prey, this method has been used in very few studies (Horgan \& Barrett 1985, Raclot et al. 1998, Dahl et al. 2003). In our work, care was taken to consider separately the WE and TAG compositions of Puffinus tenuirostris stomach oils to avoid confusion in the trophic signals from different lipid sources. Unfortunately, data available in the literature are, to a large extent, limited to total fatty alcohol and fatty acid patterns. If this has no consequence with the fatty alcohols, which can only be attributed to the WE fraction, it increases the noise to the signal ratio of the fatty acid information. To overcome this difficulty, we chose to consider only those prey species with more than $60 \%$ of either WE or TAG for integration in the prey databases. Hence, the fatty acid patterns can be related to 1 group of neutral lipids, with limited influence of the PL fraction. As shown by Mayzaud et al. (2000, 2003) from field data and by Jobling \& Bendiksen (2003) from experiments on fish, a large percentage of the variability in total fatty acid composition can usually be assigned to that of neutral lipid classes

\section{On which prey species did short-tailed shearwaters feed during long trips?}

Our results on lipid class composition of stomach oils suggested that all birds of our study had preyed on WE-rich prey species and 5 of these birds had probably preyed, in addition, on TAG-rich prey species (see above). Comparisons of WE patterns of fatty alcohols and fatty acids with LDA yielded some interesting features. The patterns of fatty alcohols and fatty acids of stomach WE from Puffinus tenuirostris differed highly from corresponding patterns in various zooplankton species (Calanoides acutus, Euchirella rostromagna, Euphausia crystallorophias, Paraeuchaeta antarctica, Rhincalanus gigas and Thysanoessa macrura). Hence, none of these species extensively contributed to the formation of stomach oils. On the contrary, the fatty alcohol of 3 myctophid fish species (Gymnoscopelus braueri, Krefftichthys anderssoni and Electrona antarctica) showed a high probability of resemblance with signatures recorded in stomach oils. This conclusion is moreover supported by the fatty acid signature of oil WE.

Comparisons of TAG fatty acid patterns have shown a strong similarity between the squid Moroteuthis ingens and the myctophid Electrona carlsbergi, suggesting a potential trophic link. The profile of $M$. ingens is that of its digestive gland, which is rich in lipids of dietary origin (Phillips et al. 2002). In that context, it is important to emphasize that $M$. ingens mainly prey upon myctophids (Cherel \& Duhamel 2003), and that its digestive gland lipid has a myctophid signature (Phillips et al. 2001, 2003). In addition, the biological cycle of $M$. ingens makes it largely unavailable to shearwaters (it was rarely eaten; Weimerskirch \& Cherel 1998), the species being mainly targeted as juveniles by penguins and as adults by large Procellariiformes and marine mammals (Cherel \& Weimerskirch 1999). Hence, the similarity between bird stomach oils and digestive gland of $M$. ingens can be seen either as an indication that shearwaters fed on squids or on prey of squids, i.e. myctophid fish (including $E$. carlsbergi). Comparisons of TAG fatty acid patterns also indicated that the ecologically important Antarctic Euphausia superba was not the major prey species participating to the formation of shearwater stomach oils contrary to the hypothesis developed by Kerry et al. (1983).

The main limitation in the use of literature data is that it is difficult to assess the validity of published results. Within a given prey species, homogeneity can be verified using multivariate analyses but little can be achieved when conflicting results are available. An example is provided with the data for the euphausiid Nyctiphanes australis, which has been reported as a major prey item of Puffinus tenuirostris (Bishop et al. 1983). Indeed, high levels of WE were reported by Bishop et al. (1983), while Cheah \& Hansen (1970a) and Virtue et al. (1995) reported a dominance of TAG without any WE. At this stage, it is impossible to ascertain whether samples were contaminated as suggested by Virtue et al. (1995) or if other reasons may explain the discrepancy. What seems unlikely however is that the same species sampled in a similar area (Tasmanian waters) would show such differences in lipid accumulation processes over years. Our dietary analysis showed that $N$. australis was a major prey during ST (during which there was no storage of stomach oil) but it was much less important in LT samples (Weimerskirch \& Cherel 1998). This, together with the fact that Tasmanian krill were barely digested in stomach contents, strongly suggests that the species was not involved in the storage of oil during LT in adult birds.

One of the major findings of our study is that biochemical analysis of lipids from stomach oil indicate that adult short-tailed shearwaters mainly prey upon sub-Antarctic and Antarctic myctophid fish during LT performed far away their breeding colony. Indeed, the patterns of fatty alcohols and fatty acids of WE both suggest that the main prey species were Krefftichthys 
anderssoni, Gymnoscopelus braueri and Electrona antarctica, and the fatty acid patterns of TAG add another myctophid species: Electrona carlsbergi. These results are in agreement with direct and indirect evidences of the feeding ecology of shearwaters during LT. First, the main component by mass of LT-food was fish, including very digested remains of various myctophids with $K$. anderssoni being the commonest (Weimerskirch \& Cherel 1998). Second, the nitrogen stable isotopic signature of shearwater plasma after a LT suggests the staple food of birds was not crustaceans but organisms feeding on crustaceans (like myctophids), and its signature is close to that of a specialist myctophid-eater, the king penguin (Y. Cherel, K. A. Hobson \& H. Weimerskirch unpubl. data).

Four species of myctophids contribute to the bulk of the myctophid biomass in the Southern Ocean (Sabourenkov 1991, Kozlov 1995), they are the WErich species Krefftichthys anderssoni and Electrona antarctica and the TAG-rich species Electrona carlsbergi and Gymnoscopelus nicholsi (Phleger et al. 1997, 1999, Lea et al. 2002). Feeding on these species indicates that short-tailed shearwaters feed for themselves in the Antarctic zone (south of the Polar Front) and the Polar Frontal Zone (between the Sub-Antarctic and the Antarctic Polar Fronts) where the species are known to occur (Hulley 1981). This is in agreement with the few satellite-tracked birds that foraged far away in the south of Australia and reached Antarctic waters during LT. Birds from Montague Island (New South Wales, Australia), took only 3 to $4 \mathrm{~d}$ to get south of $60^{\circ} \mathrm{S}$, then spent differing amounts of time at specific locations in the Southern Ocean before flying directly back to their colony (Klomp \& Schultz 2000).

\section{CONCLUSION}

Our investigations underline, for the first time, the importance of sub-Antarctic and Antarctic myctophid fish in the nutrition of adult short-tailed shearwaters during LT. They further emphasize the trophic role of myctophids in the pelagic ecosystem of the Southern Ocean, where they account for the main available biomass of macrozooplankton-eating animals for top predators (Sabourenkov 1991, Kozlov 1995). The study also stresses the need to have a reference database of fatty acid and fatty alcohol patterns of potential prey. It demonstrates the usefulness of the lipids of stomach oils of Procellariiformes as trophic markers to shed new light on predator-prey relationships and on resource acquisition and allocation processes, which are key issues in both ecosystemic and evolutionary ecology.
Acknowledgements. This study was conducted under permit no. FA96188 from the Animal Ethics Committee of the Tasmanian Parks and Wildlife Service. It would have not been possible without the help of many people in Tasmania (see Weimerskirch \& Cherel 1998). We thank Dr. P. D. Nichols, Pr. C. F. Phleger, Dr. M. M. Nelson and Dr. K. L. Phillips for the supply of detailed chromatographic data. We would also like to thank Dr. C. Thiriot, Dr. J. Dolan, Dr. V. Jones and 4 anonymous reviewers for their helpful suggestions on earlier versions of the manuscript. This research was supported by CNRS through UMR 7093 and a doctoral scholarship to M.C. from Paris VI University.

\section{LITERATURE CITED}

Ackman RG (1980) Fish lipids. In: Connell JJ (ed) Advances in fish science and technology. Fishing News Books, Surrey, p 86-103

Ackman RG (1981) Application of flame ionization detectors to thin layer chromatography on coated quartz rods. Method Enzymol 72:205-252

Ackman RG, Jangaard PM, Hoyle RJ, Brokerhoff H (1964) Origin of marine fatty acids. I. Analyses of the fatty acids produced by the diatom Skeletonema costatum. J Fish Res Board Can 21:747-756

Ackman RG, Epstein S, Eaton CA (1971) Differences in the fatty acid compositions of blubber fats from the northwestern Atlantic fin whales (Balaenoptera physalus) and harp seals (Pagophyllus groendlandica). Comp Biochem Physiol 408:683-697

Ackman RG, Hooper SN, Epstein S, Kelleher M (1972) Wax esters of Barracudia lipid: a potential replacement for sperm whale oil. J Am Oil Chem Soc 49:378-382

Albers CS, Kattner G, Hagen W (1996) The compositions of wax esters, triacylglycerols and phospholipids in Arctic and Antarctic copepods: evidence of energetic adaptations. Mar Chem 55:347-358

Auel H, Harjes M, da Rocha R, Stübing D, Hagen W (2002) Lipid biomarkers indicate different ecological niches and trophic relationships of the Arctic hyperiid amphipods Themisto abyssorum and T. libellula. Polar Biol 25: 374-383

Best NJ, Bradshaw CJA, Hindell MA, Nichols PD (2003) Vertical stratification of fatty acids in the blubber of southern elephant seals (Mirounga leonina): implications for diet analysis. Comp Biochem Physiol B 2:253-263

Bishop DG, Ritz DA, Hosie GW, Kenrick JR, Olley J (1983) Fatty acid composition of the lipids of Puffinus tenuirostris (Temminck) in relation to its diet. J Exp Mar Biol Ecol 71: $17-26$

Bligh EG, Dyer WJ (1959) A rapid method of total lipid extraction and purification. Can J Biochem Physiol 37: 911-917

Bottino NR (1975) Lipid composition of 2 species of Antarctic krill: Euphausia superba and E. crystallorophias. Comp Biochem Physiol B 50:479-484

Cheah CC, Hansen IA (1970a) Wax esters in the stomach oil of petrels. Int J Biochem 1:198-202

Cheah CC, Hansen IA (1970b) Stomach oil and tissue lipids of the petrels Puffinus pacificus and Pterodroma macroptera. Int J Biochem 1:203-208

Cherel Y, Duhamel G (2003) Diet of the squid Moroteuthis ingens (Teuthoidea: Onychoteuthidae) in the upper slope waters of the Kerguelen Islands. Mar Ecol Prog Ser 250: 197-203

Cherel Y, Weimerskirch H (1999) Spawning cycle of onycho- 
teuthid squids in the southern Indian Ocean: new information from seabird predators. Mar Ecol Prog Ser 188:93-104

Cherel Y, Bocher P, Trouvé C, Weimerskirch H (2002) Diet and feeding ecology of blue petrels Halobaena caerulea at Iles Kerguelen, Southern Indian Ocean. Mar Ecol Prog Ser 228:283-299

Clarke A (1989) Seabirds. In: Ackman RG (ed) Marine biogenic lipids, fats, and oils, Vol II. CRC Press, Boca Raton, FL, p 383-398

Clarke A, Prince PA (1976) The origin of stomach oil in marine birds: analyses of the stomach oil from six species of subAntarctic procellariiform birds. J Exp Mar Biol Ecol 23: $15-30$

Cripps GC, Atkinson A (2000) Fatty acid composition as an indicator of carnivory in Antarctic krill, Euphausia superba. Can J Fish Aquat Sci 57:31-37

Dahl TM, Lydersen C, Kovacs KM, Falk-Petersen S, Sargent J, Gjertz I, Gulliksen B (2000) Fatty acid composition of the blubber in white whales (Delphinapterus leucas). Polar Biol 23:401-409

Dahl TM, Falk-Petersen S, Gabrielsen GW, Sargent JR, Hop H, Millar RM (2003) Lipids and stable isotopes in common eider black-legged kittiwake and northern fulmar: a trophic study from an Arctic fjord. Mar Ecol Prog Ser 256: 257-269

Falk-Petersen S, Sargent JR, Lønne OJ (1999) Functional biodiversity of lipids in Antarctic zooplankton: Calanoides acutus, Calanus propinquus, Thysanoessa macrura and Euphausia crystallorophias. Polar Biol 21:37-47

Fricke H, Oehlenschläger J (1988) Fatty acid and sterol composition of the Antarctic amphipod Themisto gaudichaudii Guerin 1828. Comp Biochem Physiol B 89:39-42

Grahl-Nielsen O, Mjaavatten O (1991) Dietary influence on fatty acid composition of blubber fat of seals as determined by biopsy: a multivariate approach. Mar Biol 110: $59-64$

Hagen W, Kattner G, Graeve M (1993) Calanoides acutus and Calanus propinquus, Antarctic copepods with different lipid storage modes via wax esters or triacylglycerols. Mar Ecol Prog Ser 97:135-142

Hagen W, Kattner G, Graeve M (1995) On the lipid biochemistry of polar copepods: compositional differences in the Antarctic calanoids Euchaeta antarctica and Euchirella rostromagna. Mar Biol 123:451-457

Horgan IE, Barrett JA (1985) The use of lipid profiles in comparing the diet of seabirds. In: Siegfried WR, Condy PR, Laws RM (eds) Antarctic nutrient cycles and food webs. Springer-Verlag, Berlin, p 493-497

Hulley PA (1981) Results of the research cruises of FRV 'Walther Herwig' to South America. LVIII. Family Myctophidae (Osteichthyes, Myctophiformes). Arch Fischereiwiss 31:1-300

Iverson SJ (1993) Milk secretion in marine mammals in relation to foraging: can milk fatty acids predict diet? Symp Zool Soc Lond 66:263-291

Jobling M, Bendiksen EA (2003) Dietary lipids and temperature interact to influence tissue fatty acid compositions of Atlantic salmon, Salmo salar L., parr. Aquac Res 34: 1423-1441

Joseph JD (1975) Identification of 3, 6, 9, 12, 15-octadecapentaenoic acid in laboratory-cultured photosynthetic dinoflagellates. Lipids 10:395-403

Kattner G, Hagen W (1998) Lipid metabolism of the Antarctic euphausiid Euphausia crystallorophias and its ecological implications. Mar Ecol Prog Ser 170:203-213

Kattner G, Graeve M, Hagen W (1994) Ontogenetic and seasonal changes in lipid and fatty acid/alcohol compositions of the dominant Antarctic copepods Calanus propinquus, Calanoides acutus, Rhincalanus gigas. Mar Biol 118: 637-644

Kerry KR, Horne RSC, Dorward DF (1983) Records of the short-tailed shearwater Puffinus tenuirostris in Antarctic waters. Emu 83:35-37

Klomp NI, Schultz MA (2000) Short-tailed shearwaters breeding in Australia forage in Antarctic waters. Mar Ecol Prog Ser 194:307-310

Kozlov AN (1995) A review of the trophic role of mesopelagic fish of the Family Myctophidae in the Southern Ocean ecosystem. Comm Conserv Antarct Mar Living Resour Sci 2:71-77

Lea MA, Nichols PD, Wilson G (2002) Fatty acid composition of lipid-rich myctophids and mackerel icefish (Champsocephalus gunnari) - Southern Ocean food-web implications. Polar Biol 25:843-854

Lewis RW (1966) Studies of the glyceryl ethers of the stomach oil of Leach's petrel Oceanodroma leucorhoa (Viellot). Comp Biochem Physiol 19:363-377

Lewis RW (1969) Studies on the stomach oils of marine animals. II. Oils of some procellariiform birds. Comp Biochem Physiol 31:725-731

Marchant S, Higgins PJ (1990) Handbook of Australian, New Zealand and Antarctic birds. Oxford University Press, Melbourne

Mayzaud P, Eaton CA, Ackman RG (1976) The occurrence and distribution of octadecapentaenoic acid in a natural plankton population. A possible food chain index. Lipids 11:858-862

Mayzaud P, Virtue P, Albessard E (1999) Seasonal changes in the lipid and fatty-acid composition of the euphausiid Meganyctiphanes norvegica from the Ligurian Sea. Mar Ecol Prog Ser 186:199-210

Mayzaud P, Albessard E, Virtue P, Boutoute M (2000) Environmental constraints on the lipid structure and metabolism of euphausiids: case of Euphausia superba and Meganyctiphanes norvegica. Can J Fish Aquat Sci 57: 91-103

Mayzaud P, Boutoute M, Alonzo F (2003) Lipid composition of the Antarctic euphausiids Euphausia vallentini and Thysanoessa macrura during summer in the Indian sector of the Southern Ocean. Antarct Sci 15:463-475

Morrison WR, Smith LM (1964) Preparation of fatty acid methyl esters and dimethylacetals from lipids with boron fluoride-methanol. J Lipid Res 5:600-608

Nelson MM, Mooney BD, Nichols PD, Phleger CF (2001) Lipids of Antarctic Ocean amphipods: food chain interactions and the occurrence of novel biomarkers. Mar Chem 73:53-64

Pascal JC, Ackman RG (1976) Long chain monoethylenic alcohol and acid isomers in lipids of copepods and capelin. Chem Phys Lipids 16:219-223

Phillips KL, Jackson GD, Nichols PD (2001) Predation on myctophids by the squid Moroteuthis ingens around Macquarie and Heard Islands: stomach contents and fatty acid analyses. Mar Ecol Prog Ser 215:179-189

Phillips KL, Nichols PD, Jackson GD (2002) Lipid and fatty acid composition of the mantle and digestive gland of four Southern Ocean squid species: implications for food-web studies. Antarct Sci 14:212-220

Phillips KL, Nichols PD, Jackson GD (2003) Dietary variation of the squid Moroteuthis ingens at four sites in the Southern Ocean: stomach contents, lipid and fatty acid profiles. J Mar Biol Assoc UK 83:523-534

Phleger CF, Nichols PD, Virtue P (1997) The lipid, fatty acid and fatty alcohol composition of the myctophid fish Elec- 
trona antarctica: high level of wax esters and food-chain implications. Antarct Sci 9:258-265

Phleger CF, Nichols PD, Virtue P (1998) Lipids and trophodynamics of Antarctic zooplankton. Comp Biochem Physiol B 120:311-323

Phleger CF, Nelson MM, Mooney BD, Nichols PD (1999) Wax esters versus triacylglycerols in myctophid fishes from the Southern Ocean. Antarct Sci 11:436-444

Phleger CF, Nelson MM, Mooney BD, Nichols PD (2002) Interannual and between species comparison of the lipids, fatty acids and sterols of Antarctic krill from the US AMLR Elephant Island survey area. Comp Biochem Physiol B 131:733-747

Place AR, Stoyan NC, Ricklefs RE, Butler RG (1989) Physiological basis of stomach oil formation in Leach's stormpetrel (Oceanodroma leucorhoa). Auk 106:687-699

Raclot T, Groscolas R, Cherel Y (1998) Fatty acid evidence for the importance of myctophid fishes in the diet of king penguins, Aptenodytes patagonicus. Mar Biol 132:523-533

Reinhardt SB, Van Vleet ES (1986) Lipid composition of twenty-two species of Antarctic midwater zooplankton and fish. Mar Biol 91:149-159

Sabourenkov EN (1991) Mesopelagic fish of the Southern Ocean-summary results of recent soviet studies. Comm Conserv Antarct Mar Living Resour Sel Sci Pap 1990: 433-457

Sargent JR, Falk-Petersen S (1981) Ecological investigations on the zooplankton community in Balsfjorden, northern Norway: lipids and fatty acids in Meganyctiphanes norvegica, Thysanoessa raschi and $T$. inermis during mid-winter. Mar Biol 62:131-137

Schultz MA, Klomp NI (2000) Does the foraging strategy of adult short-tailed shearwaters cause obesity in their chicks? J Avian Biol 31:287-294

Skira IJ, Wapstra JE, Towney GN, Naarding JA (1985) Conservation of the short-tailed shearwater Puffinus tenuirostris in Tasmania, Australia. Biol Conserv 37:225-235

Smith SJ, Iverson SJ, Bowen WD (1997) Fatty acid signatures and classification trees: new tools for investigating the foraging ecology of seals. Can J Fish Aquat Sci 54:1377-1386

Virtue P, Johannes RE, Nichols PD, Young JW (1995) Bio-

Editorial responsibility: Otto Kinne (Editor-in-Chief), Oldendorf/Luhe, Germany chemical composition of Nyctiphanes australis and its possible use as an aquaculture feed source: lipids, pigments and fluoride content. Mar Biol 122:121-128

Volkman JK, Nichols PD (1991) Applications of Thin Layer Chromatography-Flame Ionization Detection to the analysis of lipids and pollutants in marine and environmental samples. J Planar Chromatogr 4:19-26

Warham J (1977) The incidence, functions and ecological significance of petrel stomach oils. Proc NZ Ecol Soc 24: 84-93

Warham J (1990) The petrels. Their ecology and breeding systems. Academic Press, London

Warham J (1996) Procellariiform lipids. In: The behaviour, population biology and physiology of the petrels. Academic Press, London, p 364-372

Warham J, Watts R, Dainty RJ (1976) The composition, energy content and function of the stomach oils of petrels (order, Procellariiformes). J Exp Mar Biol Ecol 23:1-13

Weimerskirch H, Cherel Y (1998) Feeding ecology of shorttailed shearwaters: breeding in Tasmania and foraging in the Antarctic? Mar Ecol Prog Ser 167:261-274

Weimerskirch $\mathrm{H}$, Chastel O, Ackermann L, Chaurand T, Cuenot-Chaillet F, Hindermeyer X, Judas J (1994) Alternate long and short foraging trips in pelagic seabird parents. Anim Behav 47:472-476

Weimerskirch H, Fradet G, Cherel Y (1999) Natural and experimental changes in chick provisioning in a longlived seabird, the Antarctic prion. J Avian Biol 30:165-174

Weimerskirch H, Ancel A, Calouin M, Zahariev A, Spagiari J, Kersten M, Chastel O (2003) Foraging efficiency and adjustment of energy expenditure in a pelagic seabird provisioning its chick. J Anim Ecol 72:500-508

Wilson RP (1984) An improved stomach pump for penguins and other seabirds. J Field Ornithol 55:109-112

Woodward DR, Riley MD, Buick DR, Nichols DS, Nichols PD (1995) Nutritional analysis of the flesh and oil of yolla, the Tasmanian mutton bird Puffinus tenuirostris: a useful source of omega-3 polyunsaturated fatty acids. Aust J Nutr Diet 52:87-91

Zar JH (1984) Biostatistical analysis. Prentice-Hall, Englewood Cliffs, NJ

Submitted: June 29, 2004; Accepted: December 12, 2004 Proofs received from author: March 29, 2005 